\title{
Hard exclusive processes in the backward region
}

\author{
J.P. Lansberg* \\ IPNO, Université Paris-Sud 11, CNRS/IN2P3, F-91406, Orsay, France ${ }^{\dagger}$ \\ Centre de Physique Théorique, École polytechnique, CNRS, F-91128, Palaiseau, France \\ E-mail: Jean-Philippe.Lansberg@in2p3.fr

\section{B. Pire} \\ Centre de Physique Théorique, École polytechnique, CNRS, F-91128, Palaiseau, France \\ E-mail: Bernard.Pire@cpht.polytechnique.fr
}

\section{Szymanowski}

Soltan Institute for Nuclear Studies, Warsaw, Poland

E-mail: Lech.Szymanowski@fuw.edu.pl

\begin{abstract}
We review the potentialities offered by the study of backward exclusive processes in a new scaling regime, i.e. involving a large -timelike or spacelike- $Q^{2}$ photon and a baryonic exchange in the $t$-channel. We recall the concept of Transition Distribution Amplitudes (TDAs) containing unique information on the hadron structure, then discuss how they enter the description of processes such as backward electroproduction of a pion, antiproton-proton annihilations into a dilepton + meson as well as into $J / \psi+$ meson. We then discuss first phenomenological studies for processes that are being analysed at JLAB and HERMES or that will be measured by Panda at GSI-FAIR. Finally we present outlooks for their theoretical studies based on approaches such as the pion-cloud model.
\end{abstract}

35th International Conference of High Energy Physics - ICHEP2010,

July 22-28, 2010

Paris France

\footnotetext{
*Speaker.

${ }^{\dagger}$ Permanent address at IPNO
} 


\section{Introduction}

According to a well-established framework [1], the Bjorken limit of near forward exclusive reactions with a far off-shell photon allows the factorisation of the corresponding leading-twist amplitudes into a perturbatively calculable sub-process at the quark and gluon level and non-perturbative hadronic matrix elements of light-cone non-local operators, in this case the Generalised Parton Distributions (GPDs). These are new QCD objects carrying much information on the hadronic structure. Besides, a further generalisation of the GPD concept has been proposed [2] in cases where the initial and final states are different hadronic states. When these new hadronic objects are defined through a three-quark operator (baryon-to-meson or baryon-to-photon transition), we call them baryonic Transition Distribution Amplitudes (TDAs).

In $[3,4,5]$, we have introduced the framework to study backward pion electroproduction, $\gamma^{\star}(q) N\left(p_{1}\right) \rightarrow N^{\prime}\left(p_{2}\right) \pi\left(p_{\pi}\right)$, on a proton (or neutron) target, in the Bjorken regime ( $q^{2}$ large and $q^{2} /\left(2 p_{1} . q\right)$ fixed) in terms of a factorised amplitude (see Fig. 1 (a)) where a hard part is convoluted with the aforementioned TDAs. The same framework can also be applied to the reaction, $N\left(p_{1}\right) \bar{N}\left(p_{2}\right) \rightarrow \gamma^{\star}(q) \pi\left(p_{\pi}\right)$, in the near forward region [6, 7, 8] (see Fig. 1 (b)) . In the following, we first recall the definition of the TDAs in the exemplary $p \rightarrow \pi^{0}$ case, and then discuss how they enter the description of processes such as backward electroproduction of a pion, antiproton-proton annihilations into a dilepton + meson as well as into $J / \psi+$ meson. Finally, we present some outlooks.

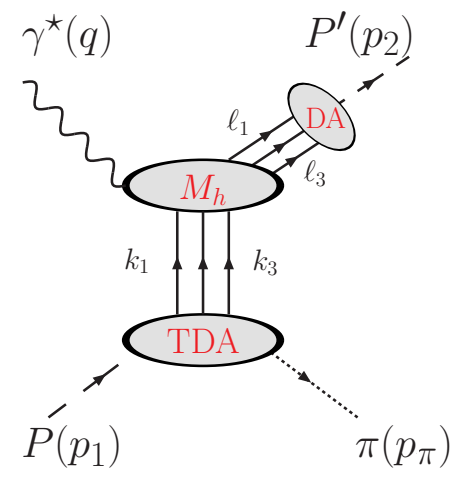

(a) $\pi^{0}$ electroproduction at small $u$

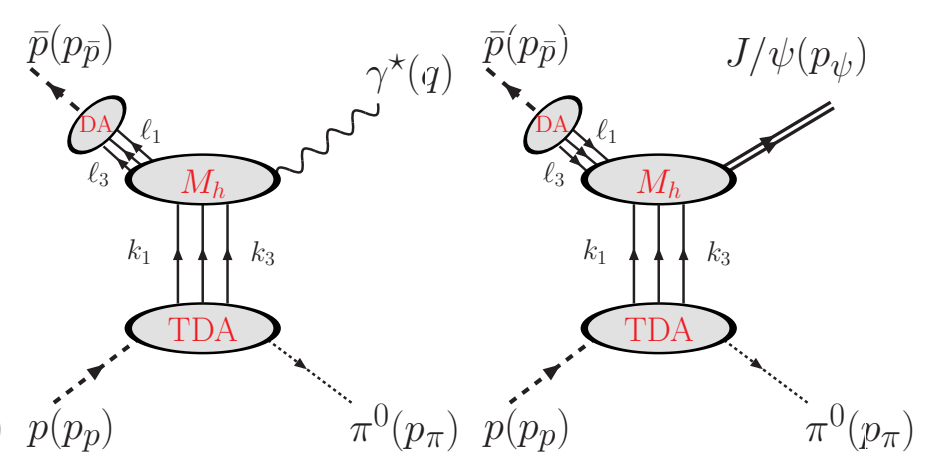

Figure 1: Illustration of the factorisation for three exclusive reactions involving the TDAs.

\section{Definition of baryonic TDAs}

The leading-twist TDAs for the $p \rightarrow \pi^{0}$ transition, $V_{i}^{p \pi^{0}}\left(x_{i}, \xi, \Delta^{2}\right), A_{i}^{p \pi^{0}}\left(x_{i}, \xi, \Delta^{2}\right)$ and $T_{i}^{p \pi^{0}}\left(x_{i}, \xi, \Delta^{2}\right)$ are defined ${ }^{1}$ as :

$$
\begin{aligned}
& 4 \mathcal{F}\left(\left\langle\pi^{0}\left(p_{\pi}\right)\left|\epsilon^{i j k} u_{\alpha}^{i}\left(z_{1} n\right) u_{\beta}^{j}\left(z_{2} n\right) d_{\gamma}^{k}\left(z_{3} n\right)\right| P\left(p_{1}\right)\right\rangle\right)=i \frac{f_{N}}{f_{\pi}}\left[V_{1}^{p \pi^{0}}(\not p C)_{\alpha \beta}\left(N^{+}\right)_{\gamma}+A_{1}^{p \pi^{0}}\left(\not p \gamma^{5} C\right)_{\alpha \beta}\left(\gamma^{5} N^{+}\right)_{\gamma}\right. \\
& +T_{1}^{p \pi^{0}}\left(\sigma_{p \mu} C\right)_{\alpha \beta}\left(\gamma^{\mu} N^{+}\right)_{\gamma}+M^{-1} V_{2}^{p \pi^{0}}(\not p C)_{\alpha \beta}\left(\Delta_{T} N^{+}\right)_{\gamma}+M^{-1} A_{2}^{p \pi^{0}}\left(\not p \gamma^{5} C\right)_{\alpha \beta}\left(\gamma^{5} \Delta_{T} N^{+}\right)_{\gamma} \\
& \left.+M^{-1} T_{2}^{p \pi^{0}}\left(\sigma_{p \Delta_{T}} C\right)_{\alpha \beta}\left(N^{+}\right)_{\gamma}+M^{-1} T_{3}^{p \pi^{0}}\left(\sigma_{p \mu} C\right)_{\alpha \beta}\left(\sigma^{\mu \Delta_{T}} N^{+}\right)_{\gamma}+M^{-2} T_{4}^{p \pi^{0}}\left(\sigma_{p \Delta_{T}} C\right)_{\alpha \beta}\left(\Delta_{T} N^{+}\right)_{\gamma}\right],
\end{aligned}
$$

\footnotetext{
${ }^{1}$ The present definitions differ from these of [3] by constant multiplicative factors and by the definition of $\sigma^{\mu \nu}$.
} 
where ${ }^{2} \sigma^{\mu \nu}=1 / 2\left[\gamma^{\mu}, \gamma^{v}\right]$ with $\sigma^{p \mu}=p_{\nu} \sigma^{\nu \mu}, \ldots, C$ is the charge conjugation matrix and $N^{+}$is the large component of the nucleon spinor $(N=(\eta \not p+\not \eta \eta)) N=N^{-}+N^{+}$with $N^{+} \sim \sqrt{p_{1}^{+}}$and $N^{-} \sim$ $\sqrt{1 / p_{1}^{+}}$). The TDAs $V_{i}, A_{i}$ and $T_{i}$ are dimensionless. Note that the first three terms in (2.1) are the only ones surviving the limit $\Delta_{T} \rightarrow 0$.

Baryonic TDAs are matrix elements of the same operator that appears in baryonic Distribution Amplitudes (DAs). The known evolution equations of this operator lead to derive evolution equations which have different forms in different regions ; one defines one ERBL-like and two types of DGLAP-like regions much in the same spirit as in the GPD case, so that the evolution equations in momentum space depend on the signs of the quark momentum fractions $x_{i}$. As for DAs, an asymptotic solution for this evolution equation exists but the phenomenological study of electromagnetic form factors leads us to strongly doubt that it is of any phenomenological relevance. We thus do not propose to take an asymptotic TDA as a realistic input for phenomenology.

On the other hand, there exists an interesting soft limit [5] when the emerging pion momentum is small, which allows one to relate proton $\rightarrow$ pion TDAs to proton DAs. The well-known soft pion theorems indeed gives:

$$
\left\langle\pi^{a}\left(p_{\pi}\right)|O| P\left(p_{1}, s_{1}\right)\right\rangle \rightarrow-\frac{i}{f_{\pi}}\left\langle 0\left|\left[Q_{5}^{a}, O\right]\right| P(p, s)\right\rangle
$$

when $\left|\vec{p}_{\pi}\right| \rightarrow 0$. One then gets very simple relations between the nucleon DAs $A^{p}, V^{p}$ and $T^{p}$ on the one hand and the $p \rightarrow \pi^{0}$ TDAs $V_{1}^{p \pi^{0}}, A_{1}^{p \pi^{0}}$ and $T_{1}^{p \pi^{0}}$ on the other hand. To get theoretical insights on the TDAs away from $\left|\vec{p}_{\pi}\right| \rightarrow 0\left(\xi \rightarrow 1\right.$ if one neglects $\left.m_{\pi}\right)$, one has to resort to general arguments such as their spectral representation [9] or to use models which successfully describe other hadronic observables [10]. Let us finally mention that, for the time being, there exists no modelling of the proton to photon TDAs, entering the description of backward DVCS [11].

\section{Processes involving the TDAs and the experimental situation}

As we have mentioned above, $p \rightarrow \pi$ baryonic TDAs appear in the description of backward electroproduction of a pion on a proton target (see Fig. 1 (a)). In terms of angles, in the $\gamma^{\star} p$ center of momentum (CM) frame, the angle between the $\gamma^{\star}$ and the pion, $\theta_{\pi}^{\star}$, is close to $180^{\circ}$. We then have $|u| \ll s$ and $t \simeq-\left(s+Q^{2}\right)$, in contrast to the fixed angle regime $u \simeq t \simeq-\left(s+Q^{2}\right) / 2\left(\theta_{\pi}^{\star} \simeq 90^{\circ}\right)$ and the forward (GPD) one $|t| \ll s$ and $u \simeq-\left(s+Q^{2}\right)\left(\theta_{\pi}^{\star} \simeq 0^{\circ}\right)$.

The TDAs appear also in similar electroproduction processes such as ep $\rightarrow e\left(p, \Delta^{+}\right)\left(\eta, \rho^{0}\right)$, $e p \rightarrow e(n, \Delta)\left(\pi^{+}, \rho^{+}\right), e p \rightarrow e \Delta^{++}\left(\pi^{-}, \rho^{-}\right)$. These processes have already been analysed, at backward angles, at JLab in the resonance region, i.e. $\sqrt{s_{\gamma^{\star} p}}=W<1.8 \mathrm{GeV}$, in order to study the

${ }^{2}$ We use the notation $\mathcal{F} \equiv(p . n)^{3} \int_{-\infty}^{\infty} \Pi_{j} d z_{j} /(2 \pi)^{3} e^{i \Sigma_{k} x_{k} z_{k} p \cdot n}$. The momenta of the process $\gamma^{\star} P \rightarrow P^{\prime} \pi$ are defined as in Fig. 1 (a). The $z$-axis is chosen along the initial nucleon and the virtual photon momenta and the $x-z$ plane is identified with the collision or hadronic plane. Then, we define the light-cone vectors $p$ and $n\left(p^{2}=n^{2}=0\right)$ such that $2 p . n=1$, as well as $P=\frac{1}{2}\left(p_{1}+p_{\pi}\right), \Delta=p_{\pi}-p_{1}$ and its transverse component $\Delta_{T}\left(\Delta_{T} \cdot \Delta_{T}=\Delta_{T}^{2}<0\right)$. From these, we define $\xi$ in an usual way as $\xi=-\frac{\Delta . n}{2 P . n}$. Expressing the momenta of the particles through their Sudakov decomposition and, keeping the firstorder corrections in the masses and $\Delta_{T}^{2}$, we have: $p_{1}=(1+\xi) p+\frac{M^{2}}{1+\xi} n, q \simeq-2 \xi\left(1+\frac{\left(\Delta_{T}^{2}-M^{2}\right)}{Q^{2}}\right) p+\frac{Q^{2}}{2 \xi\left(1+\frac{\left(\Delta_{T}^{2}-M^{2}\right)}{Q^{2}}\right)} n, p_{\pi}=$ $(1-\xi) p+\frac{m_{\pi}^{2}-\Delta_{T}^{2}}{1-\xi} n+\Delta_{T}, \Delta=-2 \xi p+\left[\frac{m_{\pi}^{2}-\Delta_{T}^{2}}{1-\xi}-\frac{M^{2}}{1+\xi}\right] n+\Delta_{T}, p_{2} \simeq-2 \xi \frac{\left(\Delta_{T}^{2}-M^{2}\right)}{Q^{2}} p+\left[\frac{Q^{2}}{2 \xi\left(1+\frac{\left(\Delta_{T}^{2}-M^{2}\right)}{Q^{2}}\right)}-\frac{m_{\pi}^{2}-\Delta_{T}^{2}}{1-\xi}+\frac{M^{2}}{1+\xi}\right] n-\Delta_{T}$. 
baryonic transition form factors in the $\pi$ channel [12] or in the $\eta$ channel [13, 14]. Data is being extracted in some channels above the resonance region. The number of events seems large enough to expect to get cross section measurements for $\left|\Delta_{T}^{2}\right|<1 \mathrm{GeV}^{2}$, which is the region described in terms of TDAs. Hermes analysis [15] for forward electroproduction may also be extended to larger values of $-t$. It has to be noted though, that present studies are limited to $Q^{2}$ of order a few $\mathrm{GeV}^{2}$, which gives no guarantee to reach the TDA regime yet. Higher- $Q^{2}$ data may be obtained at JLab-12 $\mathrm{GeV}$ and in muoproduction at Compass within the next few years.

Crossed reactions in proton-antiproton annihilation (e.g. with PANDA at GSI-FAIR [8]), with time-like photons (i.e. di-leptons) and a pion (see Fig. 1 (b)) also involve TDAs both for small $t=\left(p_{p}-p_{\pi^{0}}\right)^{2}$ and $u=\left(p_{\bar{p}}-p_{\pi^{0}}\right)^{2}$. In the latter case, the TDAs for a transition between the antiproton and the pion are probed. One can also study similar reactions with other mesons than a pion, e.g. $\bar{p} p \rightarrow \gamma^{\star}\left(\eta, \rho^{0}\right)$, or on a different target than proton $\bar{p} N \rightarrow \gamma^{\star} \pi$. Finally, one may consider $J / \psi$ production in association with a pion $\bar{p} p \rightarrow \psi \pi^{0}$ (see Fig. 1 (c)), which involves the same TDAs as $\bar{p} p \rightarrow \gamma^{\star} \pi^{0}$ and the backward electroproduction of a $\pi^{0}$. These studies will serve as very strong tests of the universality of the TDAs in different processes.

The first application of baryonic TDAs was centered on backward electroproduction of a pion [5]. In that case, the hard contribution, which consists in the scattering of the hard photon with three quarks, is known at the leading order. Extrapolating the limiting value of the TDAs obtained from the soft pion theorems to the large- $\xi$ region, we have obtained a first evaluation of the unpolarised cross section for backward electroproduction. This estimate, which is unfortunately reliable only in a very restricted kinematical domain (large- $\xi$ ), shows an interesting sensitivity to the underlying model for the proton DAs. This study has been extended to hard exclusive production of a $\gamma^{\star} \pi^{0}$ pair in $\bar{p} p$ annihilation at GSI-FAIR [7].

More work is needed before being able to proceed to quantitative comparisons between different TDA models as well as between theory and experiments. For the time being, model independent analyses - looking for scaling or dominance of $\sigma_{T}$ - sound more expedient. In this context, we have argued in [16] that the study of target transverse-spin asymmetry could be used as a test of the dominance of a hard parton-induced scattering in the backward region at large $Q^{2}$ rather than that of a soft baryon exchange in the $u$-channel. Such a reaction would only generate phases through final state interactions, expected to decrease for $W^{2} \gg\left(M+m_{\pi}\right)^{2}$ and large $Q^{2}$; the SSA would then be vanishing. On the contrary, in scatterings at the parton level, one expects an imaginary part to develop and to generate a SSA independently of whether $W^{2}$ and $Q^{2}$ are large or not.

\section{Conclusions}

Backward hard-exclusive reactions thus open a new window in the understanding of hadronic physics in the framework of the collinear-factorisation approach of QCD. Of course, the most important and most difficult problem to solve, in order to extract reliable and precise information on the baryon-to-meson transition from an incomplete set of observables such as cross sections and asymmetries, is to develop a realistic model for the TDAs. This is the subject of nonperturbative studies such as, e.g., lattice simulations. Three approaches -the soft pion theorem, the pion-cloud model and the spectral representation- are being explored and first cross-section evaluations in the whole kinematical domain covered by the TDA factorisation should be available soon. 
We are also hopeful that the $12 \mathrm{GeV}$ JLab upgrade and the start-up of GSI-FAIR will bring us the necessary experimental information to test the model-independent predictions of the TDA factorisation and then to check specific predictions from different TDA models, potentially connected to more fundamental quantity of the hadronic realm.

\section{Acknowledgments}

We thank S.J. Brodsky, G. Huber, V. Kubarovsky, K.J. Park, B. Pasquini, K. Semenov-TianShansky, P. Stoler and S. Wallon for useful and motivating discussions. This work is partly supported by the ANR contract BLAN07-2-191986. L.Sz. acknowledges the support by the Polish Grant N202 249235.

\section{References}

[1] D. Müller et al., Fortschr. Phys. 42, 101 (1994); X. D. Ji, Phys. Rev. Lett. 78, 610 (1997); A. V. Radyushkin, Phys. Rev. D56, 5524 (1997).

[2] L. L. Frankfurt, P. V. Pobylitsa, M. V. Polyakov and M. Strikman, Phys. Rev. D 60 (1999) 014010 [arXiv:hep-ph/9901429]; L. Frankfurt, M. V. Polyakov, M. Strikman, D. Zhalov and M. Zhalov, in Newport News 2002, Exclusive Processes at High Momentum Transfer (edited by A. Radyushkin, P. Stoler; Singapore, World Scientific, 2002, pp 361-368), arXiv:hep-ph/0211263.

[3] B. Pire and L. Szymanowski, Phys. Lett. B 622 (2005) 83 [arXiv:hep-ph/0504255].

[4] B. Pire and L. Szymanowski, PoS HEP2005 (2006) 103 [arXiv:hep-ph/0509368].

[5] J. P. Lansberg, B. Pire and L. Szymanowski, Phys. Rev. D 75 (2007) 074004 [Erratum-ibid. D 77 (2008) 019902]. Note that the differential cross-section plots are in $\mathrm{nb} / \mathrm{sr}$, not $\mathrm{pb} / \mathrm{sr}$ as indicated.

[6] B. Pire and L. Szymanowski, Phys. Rev. D 71 (2005) 111501 [arXiv:hep-ph/0411387].

[7] J. P. Lansberg, B. Pire and L. Szymanowski, Phys. Rev. D 76 (2007) 111502(R).

[8] M. F. Lutz, et al. [The PANDA Collaboration], arXiv:0903.3905 [hep-ex].

[9] B. Pire, K. Semenov-Tian-Shansky and L. Szymanowski, arXiv:1008.0721 [hep-ph].

[10] B. Pasquini, M. Pincetti and S. Boffi, Phys. Rev. D 80 (2009) 014017 [arXiv:0905.4018 [hep-ph]].

[11] J. P. Lansberg, B. Pire and L. Szymanowski, Nucl. Phys. A 782 (2007) 16 [arXiv:hep-ph/0607130].

[12] K. Park et al. [CLAS Collaboration], Phys. Rev. C 77 (2008) 015208 [arXiv:0709.1946 [nucl-ex]].

[13] C. S. Armstrong et al. [Jefferson Lab E94014 Collaboration], Phys. Rev. D 60 (1999) 052004.

[14] H. Denizli et al. [CLAS Collaboration], Phys. Rev. C 76 (2007) 015204.

[15] A. Airapetian et al. [HERMES Collaboration], Phys. Lett. B 659, 486 (2008).

[16] J. P. Lansberg, B. Pire and L. Szymanowski, arXiv:1008.3119 [hep-ph]. 